\title{
ANNOUNCEMENTS
}

\section{JULY MEETINGS}

July 1-5. Stability of Proteins-Theory \& Practice. Cambridge, U.K. Info: S. Mathias, Pafra Ltd., Biopreservation Div., 150 Science Park, Milton Rd., Cambridge CB4 4GG, U.K.

July 5-7. 3rd Int'l. Symp. on Delivery of Peptide Drugs. Amsterdam, The Netherlands. Info: H.A.H. Nauta, Centre for Biopharmaceutical Sciences, P.O. Box 9502, 2300 Leiden, The Netherlands. Tel: 31-7127-43-41.

July 8-11. Controlled Release Soc. 18th Int'l. Symp on Controlled Release of Bioreactive Materials. Amsterdam, The Netherlands. Info: Controlled Release Soc. Admin. Off., 16 Nottingham Dr., Lincolnshire, IL. 60069. Tel: 708-940-4277.

July 13-16. Ann. Mtg. of the European Soc. of Human Genetics. Leuven, Belgium. Info: J.J. Cassiman, Centre for Human Genetics, Univ. of Leuven, Herestraat B-3000, Leuven, Belgium. Tel: 32-16-21-58-60.

July 21-26. Gordon Conf. on Applied Environmental Microbiology. Colby-Sawyer College, NH. Info: Gordon Research Conf., Univ. of Rhode Island, Kingston, RI 02881-0801.

July 24-26. 10th Summer Symp. in Molecular Biology: Molecular Interactions in Plant Devel. University Park, PA. Info: B. LaPorte, Program Coord., 10th Summer Symp. in Molec. Biol., Penn State Univ., 329 S. Frear Lab., University Park, PA 16802. Tel: 814863-3696.

July 26-28. 1st Int'l. Conf. on Molecular Biology of Bacillus Thuringiensis. San Francisco, CA. Info: A.I. Aronson, Dept. of Biological Sciences, Purdue Univ., W. Lafayette, IN 47907. Tel: 317-494-4992.

July 28-31. Int'l. Symp. on Calcitonin GeneRelated Peptide. Graz, Austria. Info: Conf. Dept., N.Y. Academy of Sciences, 2 E. 63rd St., New York, NY 10021. Tel: 212-838-0230.

July 28-Aug. 1. 43rd Nat'l. Mtg., Amer. Assoc. for Clinical Chemistry. Washington, D.C. Info: D. Woodcock, Dir. of Mtgs., Amer. Assoc. for Clinical Chemistry, $2029 \mathrm{~K}$ St., N.W., Ste. 700, Washington, D.C. 20006. Tel: 800-892-1400

\section{AUGUST MEETINGS}

Aug. 1-4. Frontiers of Biotechnology in Agriculture '91. Sea of Galilee, Israel. Info: A. Altman, P.O. Box 5006, 61500 Tel Aviv, Israel. Tel: 972-3-654571.

5373.

Aug. 5-8. Symp. on Applic. of Biotech. to Tree Culture Protection and Utilization. Columbus, Ohio. Info: R. Cobourn, USDA Forest Service, Northeastern Forest Experiment Sta., 359 Main Rd., Delaware, $\mathrm{OH}$ 43015. Tel: 614-369-4476.

Aug. 12-15. 105th Ann. Mtng. \& Exposition-Assoc. of Official Analytical Chemists. Phoenix, AZ. Info: M. Ridgell, AOAC, 2200 Wilson Blvd., Ste. 400, Arlington, VA 222013301. Tel: 703-522-3032.

\section{COURSES AND WORKSHOPS}

June 17-21. Tumor \& normal cells (also Recombinant DNA \& PCR for diagnosis of microbial \& neoplastic disease-clin. biology II). Washington, D.C. Info: Off. Manager, CATCMB / 103 McCort-Ward, Catholic Univ. of Amer., 620 Michigan Ave., NE, Washington, D.C. 20064. Tel: 202-319-5276.

June 18-19. Clinical diagnosis using PCR \& hybridization analysis. San Diego, CA. Info: S.L. Chance, Biotech. Training Prog., 301 Main St., Ste. 3, Ames, IA 50010. Tel: 515232-8306

June 18-19a. Molecular biologic advances in cancer research, treatment, and diagnosis. Chicago, IL. Info: Dept. of Contin. Educ., Rush-Presbyterian-St. Luke's Medical Ctr., 600 S. Paulina, Chicago, IL. 60612. Tel: $312-$ 942-7095.

June 18-21. Basic cloning techniques (also June 24-28, Principles of flow cytometry). Colorado Springs, CO. Info: see for June 1819.

June 20-21. SIDR/RSE symp. on advancement of drug design. Glasgow, U.K. Info: A. Harvey, Strathclyde Inst. for Drug Research, 204 George St., Glasgow G1 1XW, U.K. Tel: 44-041-553-4155.

June 23-28. Techniques for DNA sequencing \& genomic screening. Bloomington, IN. Info: A. Fleming, Sch. of Contin. Studies, Owen Hall 204, Indiana Univ., Bloomington, IN 47405. Tel: 812-855-6329.

June 24-28a. In vitro toxicology (also Plant cell \& tissue culture). Washington, D.C. Info: see for June 17-21.

June 24-29. Practical intro. to genetic engineering. London. Info: H.A. White, Dept. of Biochemistry \& Molec. Biology, Univ. Coll. London, Gower St., London WC1E 6BT.

June 25-28. Fermentation methods and scaleup strategies. University Park, PA. Info: J. Shillenn, Biotechnology Inst. and Bioprocessing Resource Ctr., 519 Wartik Lab, University Park, PA 16802. Tel: 800-833-5533.

June 26, 27. Business of biotech (also Current trends \& applications of HPLC). New Brunswick, NJ. Info: J. Robles, Cook College Off. of Continuing Prof. Ed., Rutgers Univ., P.O. Box 231, New Brunswick, NJ 80903. Tel: 908-932-9271.

July 14. Tumour biology. Cambridge, U.K. Info: P. Whitfield, Cambridge Programme for Industry, Univ. of Cambridge, Dept. of Engineering, Trumpington St., Cambridge CB2 1PZ, U.K. Tel: 44-223-332-712.

July 1-6. Protein sequencing \& peptide synthesis (also July 8-12, Protein \& nucleic acid separation techniques; July 22-26, Immunochemistry). Washington, D.C. Info: see for June 17-21.

July 8. DNA amplification by PCR. New York (also July 22, St. Louis, MO). Info: see for June 18-19.

July 9-12. Basic cloning techniques. New York (also July 23-26, Northfield, MN). Info: see for June 18-19.

July 10. Electrochemical Detection Methods for HPLC. New Brunswick, NJ. Info: see for June $26 \& 27$
July 11-12. Controlled Release Soc. wkshp.: Regulatory affairs concerning controlled release syst. Amsterdam, The Netherlands. Info: Controlled Release Soc. Admin. Off., 16 Nottingham Dr., Lincolnshire, IL 60069. Tel: 708-940-4277.

July 22-23 (also Aug. 5-6). Basics of packed \& capillary GC. Pittsburgh, PA. Info: PACS, 409 Meade Dr., Coraopolis, PA 15108. Tel: 800-367-2587.

July 23-26. RFL.P analysis. St. Louis, MO. Info: see for June 18-19.

July 24 (also Aug. 7). Intro. to mass spectrometry. Pittsburgh, PA. Info: see for July 22-23.

July 24-26 (also Aug. 7-9) . Mass spectrometry \& mass spectral interpretation. Pittsburgh, PA. Info: see for July 22-23.

July 29-30 (also Aug. 1-2). Supercritical fluid chromatography/SFE analysis. Pittsburgh, PA. Info: see for July 22-23.

July 29-31 (also Aug. 5-7). Infrared spectrometry \& infrared spectral interpretation. Pittsburgh, PA. Info: see for July 22-23.

July 29-31a (also Aug. 7-9). Atomic Spectroscopy AA/ICP/DCP. Pittsburgh, PA. Info: see for July 22-23.

Aug. 1-2. Near IR analysis (also 2-D NMR analysis; Basics of HPLC). Pittsburgh, PA. Info: see for July 22-23

Aug. 3. Capillary electrophoresis. Pittsburgh, PA. Info: see for July 22-23.

Aug. 4. Basics of thin-layer chromatography. Pittsburgh, PA. Info: see for July 22-23

Aug. 6-9. Basic cloning techniques. Philadelphia, PA. (also Aug. 13-16, Madison, WI; Aug. 27-30, Cleveland, OH.) Info: see for June 18-19.

\section{EXECUTIVE CHANGES}

Sibia (La Jolla, CA) appointed William Comer president and CEO.

Cellcor's (Newton, MA) new CEO is Richard D'Antoni.

Biometrak (Cambridge, MA) welcomes Patrick Connoy as president and CEO.

Massachusetts Biotechnology Research Inst. (Worcester, MA) named Marc Goldberg as president and CEO.

MGI Pharma (Minneapolis, MN) announced that Dorene Kronke has joined the company as VP, sales and marketing.

Agouron Pharmaceuticals (La Jolla, CA) has appointed Susan Harris director of regulatory affairs.

Geritech (Northvalc, NJ) has chosen David Neer as VP, corporate development.

Agracetus (Middleton, WI) named James Timmin as director, new product development \& regulatory affairs. 